\title{
GW190521 Mass Gap Event and the Primordial Black Hole Scenario
}

\author{
V. De Luca $\odot,{ }^{1}$ V. Desjacques, ${ }^{2}$ G. Franciolini $\odot,{ }^{1}$ P. Pani, ${ }^{3,4}$ and A. Riotto ${ }^{1}$ \\ ${ }^{1}$ Département de Physique Théorique and Centre for Astroparticle Physics (CAP), Université de Genève, \\ 24 quai E. Ansermet, CH-1211 Geneva, Switzerland \\ ${ }^{2}$ Physics department and Asher Space Science Institute, Technion, Haifa 3200003, Israel \\ ${ }^{3}$ Dipartimento di Fisica, Sapienza Universit di Roma, Piazzale Aldo Moro 5, 00185 Roma, Italy \\ ${ }^{4}$ INFN, Sezione di Roma, Piazzale Aldo Moro 2, 00185 Roma, Italy
}

(Received 9 September 2020; accepted 12 January 2021; published 2 February 2021)

\begin{abstract}
The LIGO/Virgo Collaboration has recently observed GW190521, the first binary black hole merger with at least the primary component mass in the mass gap predicted by the pair-instability supernova theory. This observation disfavors the standard stellar-origin formation scenario for the heavier black hole, motivating alternative hypotheses. We show that GW190521 cannot be explained within the primordial black hole (PBH) scenario if PBHs do not accrete during their cosmological evolution, since this would require an abundance which is already in tension with current constraints. On the other hand, GW190521 may have a primordial origin if PBHs accrete efficiently before the reionization epoch.
\end{abstract}

DOI: 10.1103/PhysRevLett.126.051101

Introduction.-The observation of gravitational waves (GWs) from black-hole $(\mathrm{BH})$ mergers detected by the LIGO/Virgo Collaboration during the first observation runs (O1-O2-O3) [1-3] have resumed the interest in understanding the origin of the merging $\mathrm{BH}$ population [4]. A fascinating possibility is represented by $\mathrm{PBHs}$, whose formation took place in the early stages of the universe (see Ref. [5] for a review), and that can comprise a significant fraction $f_{\mathrm{PBH}}$ of the dark matter (DM) [6,7]. The possible primordial origin of at least some of the GW events has motivated several studies on the confrontation between the PBH scenario and the GW data [6-15].

The recent release of GW190521 [16], describing the coalescence of two BHs with masses $M_{1}=85_{-14}^{+21} M_{\odot}$ and $M_{2}=66_{-18}^{+17} M_{\odot}$, has assigned to PBHs even more relevance due to the so-called mass gap expected between about $65 M_{\odot}$ and $135 M_{\odot}$ in the spectrum of population I/II stellar-origin BHs. Pulsational pair instability (originated from unstable stellar cores due to the production of electron-positron pairs at high temperature) makes stars with a helium core mass in the range $\sim(32-64) M_{\odot}$ unstable. However, stars with mass above $\gtrsim 200 M_{\odot}$ and very low metallicity (typical of population III) can avoid disruption and form a population of intermediate mass BHs with masses above $\sim 135 M_{\odot}$ [17-26]. It is not excluded that BHs within the mass gap might have an astrophysical origin due to hierarchical coalescences of smaller BHs [27-33], via direct collapse of a stellar merger between an evolved star and a main sequence companion [34,35], by rapid mass accretion in primordial dense clusters [36], or by beyond standard model physics that reduces the pair instability [37].

To understand if GW190521 may have a primordial origin, one has to assess whether the corresponding merger rate is in agreement with observations and with the corresponding value of $f_{\mathrm{PBH}}$ allowed by the current experimental constraints [38]. We will perform our analysis for two scenarios: (i) the PBH mass function is determined only by the event GW190521; (ii) the PBH mass function is determined along the lines of Ref. [39], i.e., assuming all the detections by LIGO/Virgo (including GW190521) are originated by PBH mergers.

PBH mass function.-Several models have been proposed to describe how PBHs form. One of the most likely scenarios is based on the collapse of sizable overdensities in the radiation dominated epoch [40-44]. The characteristic properties of the density perturbations (arising from curvature perturbations generated during inflation) are imprinted in the PBH mass distribution, whose shape, at formation redshift $z_{i}$, is often parametrized by a log-normal function

$$
\psi\left(M, z_{i}\right)=\frac{1}{\sqrt{2 \pi} \sigma M} \exp \left(-\frac{\log ^{2}\left(M / M_{*}\right)}{2 \sigma^{2}}\right),
$$

in terms of its central mass $M_{*}$ and width $\sigma$, arising from a symmetric peak in the curvature power spectrum $[45,46]$.

If accretion is inefficient, the mass function in Eq. (1) describes the $\mathrm{PBH}$ population at the time of merger. In the opposite case, however, such mass function must be properly evolved [47-50]. Indeed, $\mathrm{PBHs}$ in binaries may be affected by periods of baryonic mass accretion for masses larger than $\mathcal{O}(10) M_{\odot}$, in which the whole binary system is responsible for gas infall provided its corresponding Bondi radius is bigger than the typical size of the binary [39,47]. This implies that both PBHs experience gas accretion with individual mass variations as $[39,47]$ 
$\dot{M}_{1}=\dot{M}_{\text {bin }} \frac{1}{\sqrt{2(1+q)}}, \quad \dot{M}_{2}=\dot{M}_{\text {bin }} \frac{\sqrt{q}}{\sqrt{2(1+q)}}$,

depending on the mass ratio $q \equiv M_{2} / M_{1} \leq 1$ and the binary's Bondi-Hoyle mass accretion rate

$$
\dot{M}_{\text {bin }}=4 \pi \lambda m_{H} n_{\text {gas }} v_{\text {eff }}^{-3} M_{\text {tot }}^{2},
$$

in terms of the binary effective velocity $v_{\text {eff }}$ relative to the baryons with cosmic mean density $n_{\text {gas }}$ and the hydrogen mass $m_{H}$. The accretion parameter $\lambda$ keeps into account the effects of gas viscosity, Hubble expansion, and the coupling of the $\mathrm{CMB}$ radiation to the gas through Compton scattering [48]. Current limits on the fraction of the DM comprised by PBHs, with masses larger than $\mathcal{O}\left(M_{\odot}\right)$, indicate that an additional DM halo is expected to form around the compact systems, acting as a catalyst that enhances the gas accretion rate $[49,51,52]$. This effect is included in the parameter $\lambda$ [47]. We account for the sharp decrease in the accretion efficiency around the epoch of structure formation $[9,11,53]$ and for other uncertainties in the model (such as x-ray preheating [54], details of the structure formation and feedbacks of local, global [49,55], and mechanical type [56]) by setting a cutoff redshift $z_{\text {cutoff }}=10$ after which we neglect accretion. Based on previous detailed investigation $[39,47]$, we consider $z_{\text {cutoff }}=10$ as a benchmark case to study the impact of accretion on the PBH scenario. Our conclusions are not qualitatively affected by this choice. In Fig. 1 we plot the evolution of the PBH masses for GW190521 with and without accretion.

The presence of accretion induces several effects in the PBH population. First, it shifts the mass distribution to higher masses and enhances its high-mass tail [47]. Second, it modifies in a redshift-dependent fashion the overall fraction of $\mathrm{PBHs}$ in DM, scaling like $f_{\mathrm{PBH}}(z) / f_{\mathrm{PBH}}\left(z_{i}\right) \sim$ $\langle M(z)\rangle /\left\langle M\left(z_{i}\right)\right\rangle$ for sufficiently small initial abundances [57]. Third, the PBH mass evolution generates a mass ratio distribution which is skewed toward $q \lesssim 1$ with a width

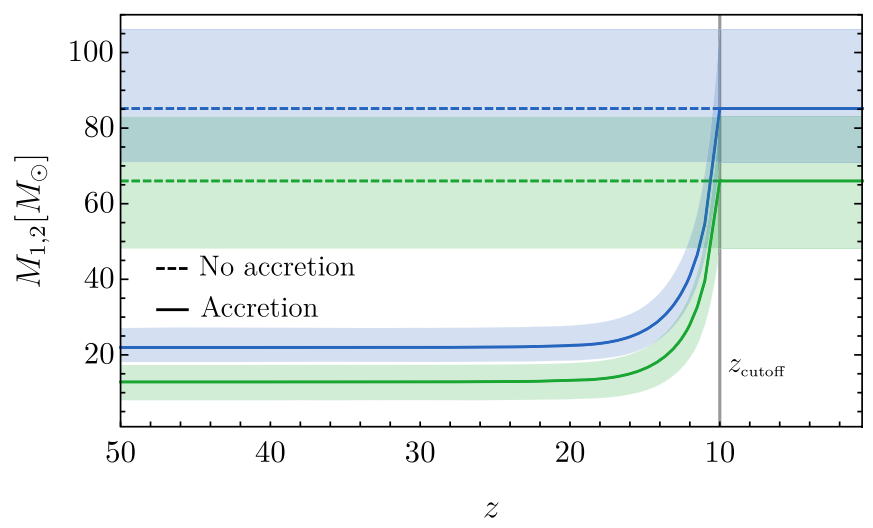

FIG. 1. Evolution of the PBH masses for GW190521 without and with accretion $\left(z_{\text {cutoff }}=10\right)$. Green (blue) bands correspond to the primary (secondary) PBH mass within $90 \%$ C.L. depending upon the initial PBH mass function [39]. Finally, the infalling gas may carry angular momentum which strongly affects the geometry of the accretion flow and the PBH spins [58]. Efficient angular momentum transfer is present when an accretion disk forms $[49,59,60]$, leading to a spin growth depending on the mass accretion as $\dot{\chi}_{j}=g\left(\chi_{j}\right) \dot{M}_{j} / M_{j}$, in terms of a function $g\left(\chi_{j}\right)$ of the dimensionless spin parameter $\chi_{j}=J_{j} / M_{j}^{2}$ of the $j$ th $\mathrm{BH}$ [39,61-63], up to the limit dictated by radiation effects, $\chi_{\max }=0.998[64,65]$. If accretion is not effective, the PBH would retain its initial spin which, in the standard formation scenario in radiation domination, is around the percent level or smaller [66,67].

$P B H$ merger rate.-The $\mathrm{PBH}$ abundance and mass function at the formation epoch determine the probability that a two-body system decouples from the Hubble flow in the early universe and forms a binary. Both the surrounding population of PBHs and the density perturbations generate a torque on the binary system, which impacts the initial distribution of the semimajor axis and eccentricity. The PBH differential merger rate is [10]

$$
\begin{aligned}
d R_{\mathrm{no}-\mathrm{acc}}= & \frac{1.6 \times 10^{6}}{\mathrm{Gpc}^{3} \mathrm{yr}} f_{\mathrm{PBH}}^{53 / 37}\left(z_{i}\right) \eta_{i}^{-34 / 37}\left(\frac{t}{t_{0}}\right)^{-34 / 37}\left(\frac{M_{\mathrm{tot}}^{i}}{M_{\odot}}\right)^{-32 / 37} \\
& \times S\left[M_{\mathrm{tot}}^{i}, f_{\mathrm{PBH}}\left(z_{i}\right)\right] \psi\left(M_{1}^{i}, z_{i}\right) \psi\left(M_{2}^{i}, z_{i}\right) d M_{1}^{i} d M_{2}^{i},
\end{aligned}
$$

where $\eta_{i}=\mu^{i} / M_{\mathrm{tot}}^{i}, \mu^{i}=M_{1}^{i} M_{2}^{i} / M_{\mathrm{tot}}^{i}, M_{\mathrm{tot}}^{i}=M_{1}^{i}+M_{2}^{i}$, and $t_{0}$ is the current age of the Universe. The suppression factor $S$ accounts for the possible binary disruption by the surrounding PBHs and matter density perturbations [10]. While the merger rate is not affected by fly-bys in the latetime Universe [68], there could be an additional suppression due to the disruption of binaries caused by other PBHs forming early substructures, which is effective only if $f_{\mathrm{PBH}} \gtrsim 0.1[10,12,69,70]$. However, the value of $f_{\mathrm{PBH}}$ necessary to explain GW190521 is at least 2 orders of magnitude smaller. Notice also that, for such small abundances, PBH clustering is irrelevant [71]; similarly, according to the results of $N$-body simulations [72], for $f_{\mathrm{PBH}} \lesssim z \times 10^{-4} \mathrm{PBHs}$ are not clustered and therefore the stringent bounds from CMB distortions [55,73], whose relevant physics occurs at $z=(300-600)$, may not be evaded by clustering arguments. Finally, in the LIGO/Virgo band the fraction of PBHs generated by previous mergers is $\sim \mathcal{O}\left(10^{-2} f_{\mathrm{PBH}}^{16 / 37}\right)$ and second-generation mergers are negligible $[47,74,75]$.

Accretion enhances the merger rate as [39]

$$
\begin{aligned}
d R_{\mathrm{acc}}= & d R_{\mathrm{no}-\mathrm{acc}}\left(\frac{M_{\mathrm{tot}}\left(z_{\mathrm{cutoff}}\right)}{M_{\mathrm{tot}}\left(z_{i}\right)}\right)^{9 / 37}\left(\frac{\eta\left(z_{\mathrm{cutoff}}\right)}{\eta\left(z_{i}\right)}\right)^{3 / 37} \\
& \times \exp \left[\frac{12}{37} \int_{t_{i}}^{t_{\text {cutoff }}} d t\left(\frac{\dot{M}_{\mathrm{tot}}}{M_{\mathrm{tot}}}+2 \frac{\dot{\mu}}{\mu}\right)\right]
\end{aligned}
$$


The corrective factors account for the hardening of the binary at $z<z_{\text {cutoff }}[39,76]$ and the augmented energy loss through GW emission [77,78], both due to the mass accretion of each PBHs.

An additional contribution comes from binaries formed in the late-time Universe, whose rate density is $[6,9]$

$$
\mathcal{V}_{\mathrm{LU}} \simeq 10^{-8}\left(\frac{f_{\mathrm{PBH}}}{10^{-3}}\right)^{53 / 21} \mathrm{Gpc}^{-3} \mathrm{yr}^{-1}
$$

This channel always gives a subdominant contribution with respect to the early-Universe one. Given the small abundances, PBH clustering does not increase the late-time Universe merger rate [71], which we therefore neglect.

The rate of events reads [79-81]

$\mathcal{R}=\int d z d M_{1} d M_{2} \frac{p_{\operatorname{det}}\left(z, M_{1}, M_{2}\right)}{1+z} \frac{d V_{c}(z)}{d z} \frac{d R_{(\mathrm{no}-) \mathrm{acc}}}{d M_{1} d M_{2}}$,

where $V_{c}(z)$ indicates the comoving volume per unit redshift. The factor $p_{\text {det }}\left(z, M_{1}, M_{2}\right)$ accounts for the detection probability of a binary averaged over the source orientation and as a function of the signal-to-noise ratio (SNR). We adopt the detectability threshold $\mathrm{SNR}=8$, the $\mathrm{O} 3$ noise power spectral density [81-85], and the IMRPhenomD waveform model [86]. The latter neglects precession, higher harmonics, and eccentricity, which could be relevant for GW190521. Using the IMRPhenomXPHM waveform model [87], we checked that precession and higher harmonics affect the SNR computation at percent level, which is much smaller than the waveform modeling and prior systematics for this event, leading most notably to uncertainties on the binary masses, spins, and eccentricity [88-91].

GW190521 as a single PBH event without accretion.In this scenario the mass function is only determined by the considered event. We choose the representative values $M_{*}=75 M_{\odot}$ and $\sigma=0.2$; different choices would result in a change of the merger rates only by $\mathcal{O}(1)$ factor. As the rate scales indicatively as $\mathcal{R} \propto f_{\mathrm{PBH}}^{53 / 37}$, the abundance is found to be relatively stable with respect to reasonable changes of the mass function. From Eq. (4), we estimate the abundance required to match the observed event rate which, given the current high uncertainties [16,91], we assume to be $\mathcal{R} \simeq 1 /$ yr. This yields $f_{\mathrm{PBH}} \simeq 1.2 \times 10^{-4}$. In Fig. 2 we compare this value with the constraints, showing a tension with the bounds coming from CMB distortions.

GW190521 as a single PBH event with accretion.-In this scenario the two PBHs evolve from initial masses of $M_{1}^{\mathrm{i}} \simeq 22 M_{\odot}$ and $M_{2}^{\mathrm{i}} \simeq 13 M_{\odot}$, respectively (Fig. 1). We choose a representative narrow initial mass function with $M_{*}=18.5 M_{\odot}$ and $\sigma=0.2$. The abundance corresponding to the observed merger rate is $f_{\mathrm{PBH}}\left(z_{i}\right) \simeq 2.5 \times 10^{-5}$ $\left[f_{\mathrm{PBH}}(z=0) \simeq 3.7 \times 10^{-5}\right]$, which is smaller than the one found in the previous section since accretion generally leads to an enhancement of the merger rate, see Eq. (5). As shown in Fig. 2, this value of $f_{\mathrm{PBH}}$ is allowed by the current constraints, which are strongly relaxed in the presence of accretion [57].

GW190521 as an event within the full PBH population.Finally, we perform a population-driven analysis assuming that all observations (O1/O2, GW190412, GW190815, GW190521) have a primordial origin. Following the procedure in Ref. [39], we implement a maximum-likelihood
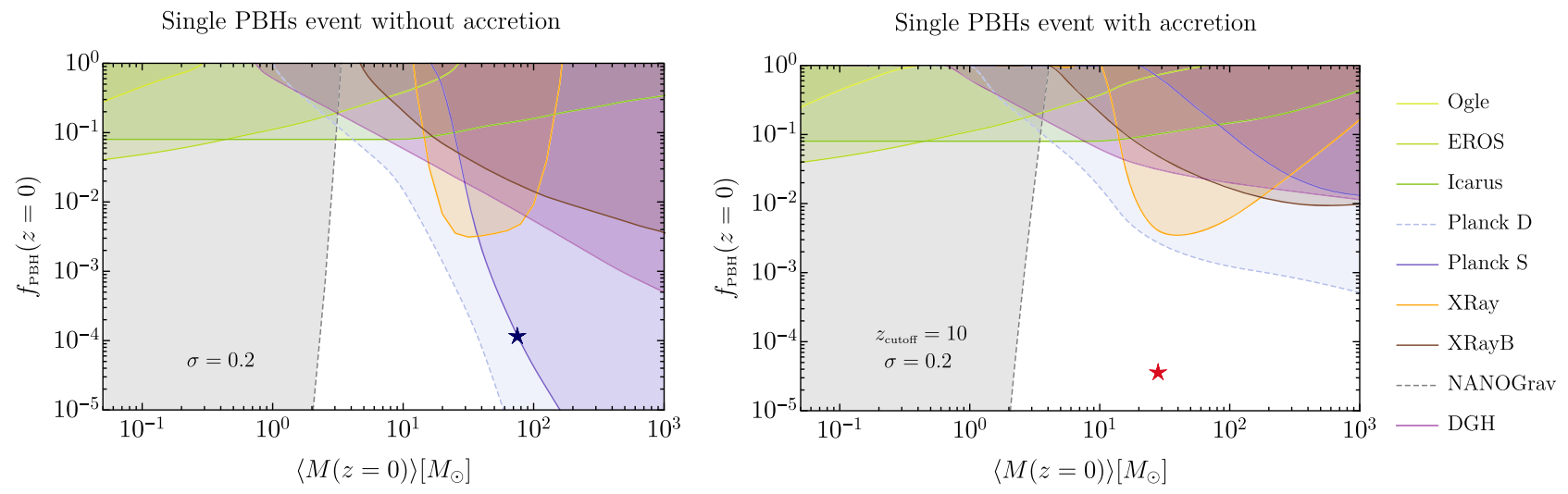

FIG. 2. Constraints on $f_{\mathrm{PBH}}$ in the first scenario. The strongest ones come from the NANOGrav experiment [92] (applicable only for PBHs formed from collapse of density perturbations and in the absence of non-Gaussianities; see Refs. [93,94]), CMB distortions (Planck D and Planck S) [55,73], x-rays (XRay) [95,96], X-ray binaries (XRayB) [97], EROS [98], Icarus [99], radio observations (Ogle) [100], and dwarf galaxy heating (DGH) [101]. Constraints on monochromatic PBH mass functions are adapted to extended mass functions with the techniques described in Refs. [46,102]. Left: The star indicates the PBH abundance needed to explain the single event GW190521 in the PBH scenario without accretion. Right: Same as in the left panel but in the scenario with accretion, accounting for the change of PBH masses and abundances as discussed in detail in Ref. [57]. In particular, early-Universe constraints NANOGrav and Planck (D/S) are computed with the high-redshift mass function and mapped to low redshift, whereas late-time Universe constraints are evaluated with the mass function evolved up to low redshift. 

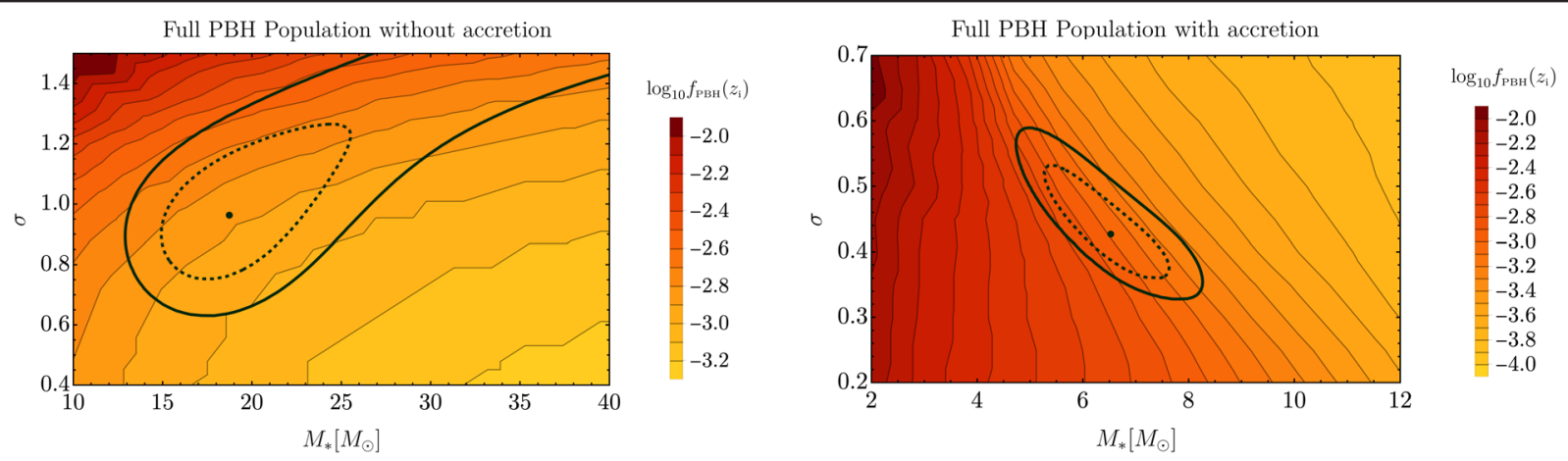

FIG. 3. Likelihood in the PBH mass-function parameter space in the second scenario. Dashed (solid) lines correspond to $1 \sigma(2 \sigma)$ contours. Left: No accretion scenario. Right: Accretion scenario with $z_{\text {cutoff }}=10$.

analysis to find the PBH mass function at formation, see Fig. 3.

In the case without relevant accretion, the best-fit values are $M_{*}=19 M_{\odot}$ and $\sigma=0.97$. The presence of the additional event GW190521 with high masses causes a preference for a broader mass function. We do not observe, however, a dramatic change in the best-fit parameters relative to those found in Ref. [39], since GW190521 is only one newly added event out of 13 observations released to date, and not particularly loud (SNR $\approx 14.7$ ) [16]. One can notice a characteristic trend for $f_{\mathrm{PBH}}$. Lighter PBHs reduce the fraction of detectable binaries. Also, for wider mass functions, the merger rate of binaries with $M_{\text {tot }} \gg M_{*}$ is suppressed [10]. Both effects require a larger $f_{\mathrm{PBH}}$ to fit the data. The shape of the $1 \sigma / 2 \sigma$ contours can be explained as follows. As $M_{*}$ moves away from its best fit, a wider mass function is needed. A lower $M_{*}$ tail is absent due to the suppression factor decreasing the merger rate for the observed masses $M_{\text {tot }} \gg M_{*}$, while a large $M_{*}-\sigma$ tail is still allowed since the suppression factor reduces the unwanted contribution of very large masses.

Fixing the merger rate to the observed one yields a value of $f_{\mathrm{PBH}}=1.3 \times 10^{-3}$, which is already ruled out by the (conservative) bound coming from Planck S, and possibly by NANOGrav (see caption of Fig. 4). The observable rate of events with masses in the mass gap or at least as extreme as GW190521 is

$$
\begin{aligned}
\mathcal{R}\left(M_{1,2}>65 M_{\odot}\right) & \simeq 7 \times 10^{-2} / \mathrm{yr}, \\
\mathcal{R}\left(M_{1}>85 M_{\odot}, \quad M_{2}>65 M_{\odot}\right) & \simeq 2 \times 10^{-2} / \mathrm{yr} .
\end{aligned}
$$

This proves GW190521 to be an outlier of the population and not generally expected in the full population scenario without accretion.

In the case with sizable accretion up to $z_{\text {cutoff }}=10$, the best-fit parameters are $M_{*}=6.5 M_{\odot}$ and $\sigma=0.43$, see Fig. 3. The contours showing the $1 \sigma / 2 \sigma$ C.I. are found to be significantly narrower relative to the no-accretion case. This is due to a stronger dependence of the binary masses and merger rates to $M_{*}$ and $\sigma$ as a result of the large impact of accretion onto binaries with total mass above $\mathcal{O}(10) M_{\odot}$. For large $M_{*}$ and $\sigma$, the merger rate is highly enhanced as in Eq. (5), leading to smaller values of $f_{\mathrm{PBH}}$, at variance with the no-accretion case. For small values of $M_{*}$, accretion gradually becomes less relevant and the behavior of $f_{\mathrm{PBH}}$ tends towards the one observed in the left panel of Fig. 3. Unlike the no-accretion case, a larger value of $M_{*}$ is correlated with a narrower mass function, since accretion broadens the initial mass distribution [57]. Also in this case, the initial mass function does not differ significantly relative to the one inferred without GW190521.

Fixing the merger rate with the LIGO/Virgo observations restrains the abundance to be $f_{\mathrm{PBH}}\left(z_{i}\right) \simeq 9.7 \times 10^{-4}$ $\left[f_{\mathrm{PBH}}(z=0) \simeq 1.0 \times 10^{-3}\right]$, which is compatible with current constraints, see Fig. 4. The merger rate of mass gap events (or as massive as GW190521) is

$$
\begin{aligned}
\mathcal{R}\left(M_{1,2}>65 M_{\odot}\right) & \simeq 1.1 / \mathrm{yr}, \\
\mathcal{R}\left(M_{1}>85 M_{\odot}, M_{2}>65 M_{\odot}\right) & \simeq 0.8 / \mathrm{yr},
\end{aligned}
$$

which are compatible with the observed rate. Therefore, in the full population scenario with accretion, GW190521 is not only perfectly allowed, but also generally expected. This conclusion is solid against modeling systematics (e.g., changes of the accretion rate) and is also conservative. Indeed, in the more realistic hypothesis in which some GW events have an astrophysical origin (e.g., those whose parameters are slightly in tension with the $\mathrm{PBH}$ scenario, but see Ref. [103] for the role of priors in alleviating this tension), removing them from the analysis would make our conclusion on the viability of GW190521 as a primordial binary even more robust.

Conclusions.-Motivated by the existence of a mass gap in the spectrum of stellar-origin BHs, we have conducted an in-depth analysis to understand if the recently observed event GW190521 can be explained by the PBH hypothesis. We have explored two opposite scenarios: the first assumes that this event is the only one of primordial origin among the LIGO/Virgo catalogs; the second supposes that all GW 
Full PBH Population without accretion

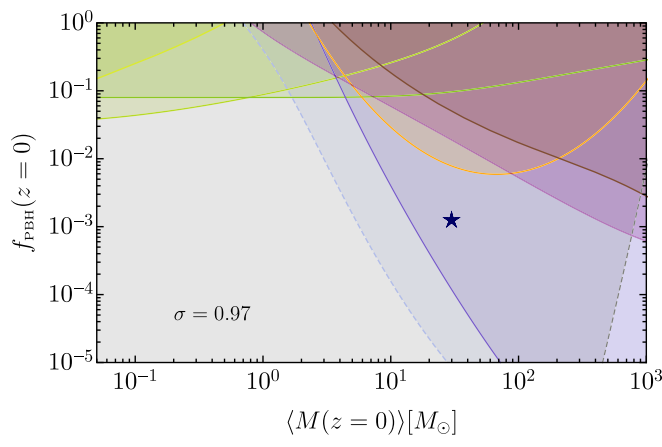

Full PBH Population with accretion

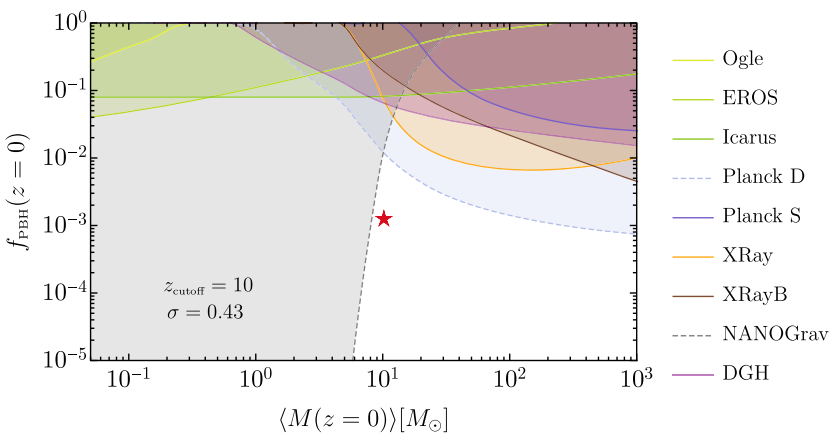

FIG. 4. Same as in Fig. 2 for the second scenario. The stars indicate $f_{\mathrm{PBH}}$ needed to explain GW190521. Notice that the NANOGrav constraint, relevant only below $M_{\odot}$ for a monochromatic population [92], is able to constrain wide enough mass functions peaked at much larger masses.

events observed so far are primordial and GW190521 is part of the inferred $\mathrm{PBH}$ population.

We found that if accretion is negligible throughout the PBH cosmological evolution and within the second hypothesis, GW190521 would be an outlier of the population with a corresponding merger rate orders of magnitude below the observed one. Furthermore, in both scenarios without accretion, the abundance required to match the merger rates is always largely in tension with the bounds coming from $\mathrm{CMB}$ distortions and, within the second hypothesis, with the one possibly coming from NANOGrav observations.

We showed, however, that accretion relaxes the tension with the upper bounds on $f_{\mathrm{PBH}}$ in both scenarios, allowing for a primordial origin of GW190521. Furthermore, mergers similar to the newly discovered mass gap event GW190521 and with a rate compatible with this observation are predicted by a PBH population inferred under the assumption that all events seen by LIGO/Virgo are primordial.

If accretion is relevant for the PBHs, the mass ratio should be close to unity and the individual spins should be nonvanishing, with the spin of the secondary component always bigger than the one of the primary [39]. Even though no firm conclusion can be drawn due to the small SNR and the large uncertainties coming from different waveform models [91], this pattern seems to be in agreement with the parameters measured for GW190521. There is evidence that the individual spins of the event are large and likely lie on the orbital plane. While sizable spins are in tension with the nonaccreting scenario, they are easily explained when accretion is efficient. Since the Bondi radii of the individual PBHs in the binary are comparable to the characteristic orbital distance, the accretion flow's geometry is complex: the orientations of individual accretion disks are independent and randomly distributed irrespective of the direction of the orbital angular momentum. Thus, the inferred values of the binary components' spins are consistent with the accreting PBH scenario.

The accretion model used in this study relies on a Newtonian approximation. General-relativistic effects might significantly increase the accretion rate in the presence of a large density contrast in the accretion flow [104]. It would be very interesting to extend numerical simulations [104] to the case under consideration, i.e., a BH binary moving in a (geometrically complex) density profile. We stress, however, that the accretion rate decreases by several orders of magnitude for PBH masses $\lesssim 10 M_{\odot}$ [49], and therefore even an order-of-magnitude increase in $\dot{M}$ does not change the conclusions drawn from Figs. 2 and 4.

Our results give strong motivation to reconsider the parameter estimation of GW190521 incorporating the correct $\mathrm{PBH}-$ motivated prior distributions to infer the binary parameters in the PBH scenario [103], and to perform a Bayesian comparison between the PBH scenario and astrophysical ones that may explain spinning binaries in the mass gap, like hierarchical mergers [27-31,33] or others $[34,35]$.

Computations were performed at University of Geneva on the Baobab cluster. We acknowledge use of the software package PYCBC [105]. V. D. L., G. F., and A. R. are supported by the Swiss National Science Foundation (SNSF), project The Non-Gaussian Universe and Cosmological Symmetries, Project No. 200020-178787. V. D. acknowledges support by the Israel Science Foundation (Grant No. 1395/16). P. P. acknowledges financial support provided under the European Union's H2020 ERC, Starting Grant Agreement No. DarkGRA-757480, and under the MIUR PRIN and FARE programmes (GW-NEXT, CUP: B84I20000100001), and support from the Amaldi Research Center funded by the MIUR program "Dipartimento di Eccellenza" (CUP: B81I18001170001).

[1] B. P. Abbott et al. (LIGO Scientific and Virgo Collaborations), Phys. Rev. X 9, 031040 (2019).

[2] R. Abbott et al. (LIGO Scientific and Virgo Collaborations), arXiv:2004.08342.

[3] R. Abbott et al. (LIGO Scientific and Virgo Collaborations) Astrophys. J. 896, L44 (2020). 
[4] L. Barack et al., Classical Quantum Gravity 36, 143001 (2019).

[5] M. Sasaki, T. Suyama, T. Tanaka, and S. Yokoyama, Classical Quantum Gravity 35, 063001 (2018).

[6] S. Bird, I. Cholis, J. B. Munoz, Y. Ali-Haïmoud, M. Kamionkowski, E. D. Kovetz, A. Raccanelli, and A. G. Riess, Phys. Rev. Lett. 116, 201301 (2016).

[7] M. Sasaki, T. Suyama, T. Tanaka, and S. Yokoyama, Phys. Rev. Lett. 117, 061101 (2016); Phys. Rev. Lett. 121, 059901(E) (2018).

[8] S. Clesse and J. Garca-Bellido, Phys. Dark Universe 15, 142 (2017).

[9] Y. Ali-Haïmoud, E. D. Kovetz, and M. Kamionkowski, Phys. Rev. D 96, 123523 (2017).

[10] M. Raidal, C. Spethmann, V. Vaskonen, and H. Veermäe, J. Cosmol. Astropart. Phys. 02 (2019) 018.

[11] G. Hütsi, M. Raidal, and H. Veermäe, Phys. Rev. D 100, 083016 (2019).

[12] V. Vaskonen and H. Veermäe, Phys. Rev. D 101, 043015 (2020).

[13] A. D. Gow, C. T. Byrnes, A. Hall, and J. A. Peacock, J. Cosmol. Astropart. Phys. 01 (2020) 031.

[14] S. Clesse and J. Garcia-Bellido, arXiv:2007.06481.

[15] A. Hall, A. D. Gow, and C. T. Byrnes, arXiv:2008.13704.

[16] R. Abbott et al. (LIGO Scientific and Virgo Collaborations), Phys. Rev. Lett. 125, 101102 (2020).

[17] G. Rakavy and G. Shaviv, Astrophys. J. 148, 803 (1967).

[18] Z. Barkat, G. Rakavy, and N. Sack, Phys. Rev. Lett. 18, 379 (1967).

[19] Gary S. Fraley, Astrophys. Space Sci. 2, 96 (1968).

[20] A. Heger and S.E. Woosley, Astrophys. J. 567, 532 (2002).

[21] S. E. Woosley, S. Blinnikov, and A. Heger, Nature (London) 450, 390 (2007).

[22] K. Belczynski, A. Heger, W. Gladysz, A. J. Ruiter, S. Woosley, G. Wiktorowicz, H. Y. Chen, T. Bulik, R. O'Shaughnessy, D. E. Holz, C. L. Fryer, and E. Berti, Astron. Astrophys. 594, A97 (2016).

[23] S. Woosley, Astrophys. J. 836, 244 (2017).

[24] S. Stevenson, M. Sampson, J. Powell, A. Vigna-Gmez, C. J. Neijssel, D. Szcsi, and I. Mandel, Astrophys. J. 882, 121 (2019).

[25] R. Farmer, M. Renzo, S. E. de Mink, P. Marchant, and S. Justham, Astrophys. J. 887, 53 (2019).

[26] M. Renzo, R. J. Farmer, S. Justham, S. E. de Mink, Y. Gtberg, and P. Marchant, Mon. Not. R. Astron. Soc. 493, 4333 (2020).

[27] M. Fishbach, D. E. Holz, and B. Farr, Astrophys. J. Lett. 840, L24 (2017).

[28] D. Gerosa and E. Berti, Phys. Rev. D 100, 041301 (2019).

[29] C. L. Rodriguez, M. Zevin, P. Amaro-Seoane, S. Chatterjee, K. Kremer, F. A. Rasio, and C. S. Ye, Phys. Rev. D 100, 043027 (2019).

[30] V. Baibhav, D. Gerosa, E. Berti, K. W. K. Wong, T. Helfer, and M. Mould, Phys. Rev. D 102, 043002 (2020).

[31] C. Kimball, C. Talbot, C. P. L. Berry, M. Carney, M. Zevin, E. Thrane, and V. Kalogera, arXiv:2005.00023.

[32] J. Samsing and K. Hotokezaka, arXiv:2006.09744.

[33] M. Mapelli, F. Santoliquido, Y. Bouffanais, M. A. Sedda, N. Giacobbo, M. C. Artale, and A. Ballone, arXiv:2007.15022.
[34] U. N. Di Carlo, M. Mapelli, Y. Bouffanais, N. Giacobbo, F. Santoliquido, A. Bressan, M. Spera, and F. Haardt, Mon. Not. R. Astron. Soc. 497, 1043 (2020).

[35] U. N. Di Carlo, M. Mapelli, N. Giacobbo, M. Spera, Y. Bouffanais, S. Rastello, F. Santoliquido, M. Pasquato, A. Ballone, A. A. Trani, S. Torniamenti, and F. Haardt, arXiv: 2004.09525.

[36] Z. Roupas and D. Kazanas, Astron. Astrophys. 632, L8 (2019).

[37] J. Sakstein, D. Croon, S. D. McDermott, M. C. Straight, and E. J. Baxter, arXiv:2009.01213.

[38] B. Carr, K. Kohri, Y. Sendouda, and J. Yokoyama, arXiv:2002.12778.

[39] V. De Luca, G. Franciolini, P. Pani, and A. Riotto, J. Cosmol. Astropart. Phys. 06 (2020) 044.

[40] S. Blinnikov, A. Dolgov, N. K. Porayko, and K. Postnov, J. Cosmol. Astropart. Phys. 11 (2016) 036.

[41] P. Ivanov, P. Naselsky, and I. Novikov, Phys. Rev. D 50, 7173 (1994).

[42] J. García-Bellido, A. D. Linde, and D. Wands, Phys. Rev. D 54, 6040 (1996).

[43] P. Ivanov, Phys. Rev. D 57, 7145 (1998).

[44] J. Garca-Bellido, J. Phys. Conf. Ser. 840, 012032 (2017).

[45] A. Dolgov and J. Silk, Phys. Rev. D 47, 4244 (1993).

[46] B. Carr, M. Raidal, T. Tenkanen, V. Vaskonen, and H. Veermäe, Phys. Rev. D 96, 023514 (2017).

[47] V. De Luca, G. Franciolini, P. Pani, and A. Riotto, J. Cosmol. Astropart. Phys. 04 (2020) 052.

[48] M. Ricotti, Astrophys. J. 662, 53 (2007).

[49] M. Ricotti, J. P. Ostriker, and K. J. Mack, Astrophys. J. 680, 829 (2008).

[50] J. R. Rice and B. Zhang, J. High Energy Astrophys. 13-14, 22 (2017).

[51] J. Adamek, C. T. Byrnes, M. Gosenca, and S. Hotchkiss, Phys. Rev. D 100, 023506 (2019).

[52] K. J. Mack, J. P. Ostriker, and M. Ricotti, Astrophys. J. 665, 1277 (2007).

[53] G. Hasinger, arXiv:2003.05150.

[54] S. Oh and Z. Haiman, Mon. Not. R. Astron. Soc. 346, 456 (2003).

[55] Y. Ali-Haïmoud and M. Kamionkowski, Phys. Rev. D 95, 043534 (2017).

[56] V. Bosch-Ramon and N. Bellomo, Astron. Astrophys. 638, A132 (2020).

[57] V. De Luca, G. Franciolini, P. Pani, and A. Riotto, arXiv: 2003.12589.

[58] E. Berti and M. Volonteri, Astrophys. J. 684, 822 (2008).

[59] N. Shakura and R. Sunyaev, Astron. Astrophys. 24, 337 (1973).

[60] I. D. Novikov and K. S. Thorne, Astrophysics and black holes, in Les Houches Summer School of Theoretical Physics: Black Holes (1973), pp. 343-550, https:// inspirehep.net/literature/1361968.

[61] J. M. Bardeen, W. H. Press, and S. A. Teukolsky, Astrophys. J. 178, 347 (1972).

[62] R. Brito, V. Cardoso, and P. Pani, Classical Quantum Gravity 32, 134001 (2015).

[63] M. Volonteri, P. Madau, E. Quataert, and M. J. Rees, Astrophys. J. 620, 69 (2005).

[64] K. S. Thorne, Astrophys. J. 191, 507 (1974). 
[65] C. F. Gammie, S. L. Shapiro, and J. C. McKinney, Astrophys. J. 602, 312 (2004).

[66] V. De Luca, V. Desjacques, G. Franciolini, A. Malhotra, and A. Riotto, J. Cosmol. Astropart. Phys. 05 (2019) 018.

[67] M. Mirbabayi, A. Gruzinov, and J. Norena, J. Cosmol. Astropart. Phys. 03 (2020) 017.

[68] S. Young and A. S. Hamers, arXiv:2006.15023.

[69] K. Jedamzik, arXiv:2006.11172.

[70] K. Jedamzik, arXiv:2007.03565.

[71] V. De Luca, V. Desjacques, G. Franciolini, and A. Riotto, J. Cosmol. Astropart. Phys. 11 (2020) 028.

[72] D. Inman and Y. Ali-Haïmoud, Phys. Rev. D 100, 083528 (2019).

[73] P. D. Serpico, V. Poulin, D. Inman, and K. Kohri, Phys. Rev. Research 2, 023204 (2020).

[74] L. Liu, Z. K. Guo, and R. G. Cai, Eur. Phys. J. C 79, 717 (2019).

[75] Y. Wu, Phys. Rev. D 101, 083008 (2020).

[76] A. Caputo, L. Sberna, A. Toubiana, S. Babak, E. Barausse, S. Marsat, and P. Pani, Astrophys. J. 892, 90 (2020).

[77] P. C. Peters and J. Mathews, Phys. Rev. 131, 435 (1963).

[78] P. C. Peters, Phys. Rev. 136, B1224 (1964).

[79] L. S. Finn and D. F. Chernoff, Phys. Rev. D 47, 2198 (1993).

[80] E. Berti, A. Buonanno, and C. M. Will, Phys. Rev. D 71, 084025 (2005).

[81] M. Dominik, E. Berti, R. O’Shaughnessy, I. Mandel, K. Belczynski, C. Fryer, D. E. Holz, T. Bulik, and F. Pannarale, Astrophys. J. 806, 263 (2015).

[82] B. Abbott et al. (KAGRA, LIGO Scientific, and VIRGO Collaborations), Living Rev. Relativity 21, 3 (2018).

[83] https://dcc.ligo.org/LIGO-T2000012/public.

[84] K. W. K. Wong, K. K. Y. Ng, and E. Berti, arXiv:2007.10350.

[85] D. Gerosa, G. Pratten, and A. Vecchio, arXiv:2007.06585.

[86] S. Khan, S. Husa, M. Hannam, F. Ohme, M. Prrer, X. Jimnez Forteza, and A. Boh, Phys. Rev. D 93, 044007 (2016).

[87] G. Pratten, C. García-Quirós, M. Colleoni, A. RamosBuades, H. Estellés, M. Mateu-Lucena, R. Jaume, M. Haney, D. Keitel, and J. E. Thompson, arXiv:2004.06503.
[88] I. M. Romero-Shaw, P. D. Lasky, E. Thrane, and J. C. Bustillo, Astrophys. J. Lett. 903, L5 (2020).

[89] V. Gayathri, J. Healy, J. Lange, B. O'Brien, M. Szczepanczyk, I. Bartos, M. Campanelli, S. Klimenko, C. Lousto, and R. O'Shaughnessy, arXiv:2009.05461.

[90] A. H. Nitz and C. D. Capano, arXiv:2010.12558.

[91] R. Abbott et al. (LIGO Scientific and Virgo Collaborations), Astrophys. J. Lett. 900, L13 (2020).

[92] Z. C. Chen, C. Yuan, and Q. G. Huang, Phys. Rev. Lett. 124, 251101 (2020).

[93] T. Nakama, J. Silk, and M. Kamionkowski, Phys. Rev. D 95, 043511 (2017).

[94] R. G. Cai, S. Pi, S. J. Wang, and X. Y. Yang, J. Cosmol. Astropart. Phys. 10 (2019) 059.

[95] D. Gaggero, G. Bertone, F. Calore, R. M. T. Connors, M. Lovell, S. Markoff, and E. Storm, Phys. Rev. Lett. 118, 241101 (2017).

[96] J. Manshanden, D. Gaggero, G. Bertone, R. M. Connors, and M. Ricotti, J. Cosmol. Astropart. Phys. 06 (2019) 026.

[97] Y. Inoue and A. Kusenko, J. Cosmol. Astropart. Phys. 10 (2017) 034.

[98] R. A. Allsman et al. (Macho Collaboration), Astrophys. J. 550, L169 (2001).

[99] M. Oguri, J. M. Diego, N. Kaiser, P. L. Kelly, and T. Broadhurst, Phys. Rev. D 97, 023518 (2018).

[100] H. Niikura, M. Takada, S. Yokoyama, T. Sumi, and S. Masaki, Phys. Rev. D 99, 083503 (2019).

[101] P. Lu, V. Takhistov, G. B. Gelmini, K. Hayashi, Y. Inoue, and A. Kusenko, arXiv:2007.02213.

[102] N. Bellomo, J. L. Bernal, A. Raccanelli, and L. Verde, J. Cosmol. Astropart. Phys. 01 (2018) 004.

[103] S. Bhagwat, V. De Luca, G. Franciolini, P. Pani, and A. Riotto, arXiv:2008.12320.

[104] A. Cruz-Osorio and L. Rezzolla, Astrophys. J. 894, 147 (2020).

[105] A. Nitz et al., gwastro/pycbc: РYCBC release v1.15.5 (2020). 